\title{
Variación del nitrógeno ureico en leche según la temporada del año y días en leche
}

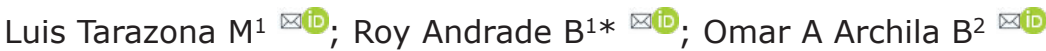

\begin{abstract}
${ }^{1}$ Universidad Pedagógica y Tecnológica de Colombia. Facultad de Ciencias Agropecuarias. Escuela de Medicina Veterinaria y Zootecnia. Laboratorio de análisis de la calidad de la leche y control de mastitis. Grupo de investigación en producción animal del trópico alto colombiano GIPATRACOL. Tunja, Boyacá, Colombia.

${ }^{2}$ Boehringer Ingelheim. Colombia.

*Correspondencia: roy.andrade@uptc.edu.co
\end{abstract}

Recibido: Mayo 2020; Aceptado: Junio 2021; Publicado: Agosto 2021.

\section{RESUMEN}

Objetivo. Determinar la variación de NUL según las temporadas del año (época de lluvia y seca) y los días en leche (DEL) de vacas lecheras de dos ganaderías en el altiplano boyacense de Colombia. Materiales y métodos. Se realizó un estudio de tipo descriptivo cuantitativo por conveniencia. Se incluyeron 4.901 muestras de leche de 112 animales ubicados a una altura de 2600 msnm durante un período de 24 meses, agrupando las muestras de acuerdo con la temporada y los días en producción de leche. Las muestras fueron procesadas a través de metodología enzimática espectofotométrica para la determinación del NUL. Se realizó un análisis de varianza para los grupos por temporada climática, mientras que para los días en leche se hizo una descripción cuantitativa. Resultados. EI NUL varió según la temporada del año $(p<0.05)$, siendo mayores los valores durante la época Iluviosa. EI NUL fue menor durante los primeros $60 \mathrm{DEL}$ en la mayoría de los grupos conformados y los picos se presentaron a partir de los 180 DEL. Los menores valores de NUL de acuerdo a los días en leche se determinaron en los primeros 60 días en la mayoría de los grupos conformados, así mismo los principales picos se determinaron a partir de los $180 \mathrm{DEL}$. Conclusiones. El NUL varió de acuerdo con la temporada siendo más alto en lluvias, el NUL fue menor durante los primeros 60 DEL en la mayoría de los grupos conformados y los picos se presentaron a partir de los $180 \mathrm{DEL}$.

Palabras clave: Vacas lecheras; proteína; balance nutricional; urea; energía (Fuentes: DeCS, CAB).

\section{ABSTRACT}

Objective. Determine the variation of MUN according to the seasons of the year (rainy and dry season) and the days in milk (DIM) of dairy cows from two herds in the Boyacá highlands of Colombia. Materials and methods. A descriptive quantitative study was carried out for convenience. 4,901 milk samples were included from 112 animals located at a height of 2,600 meters above sea level during a period of 24 months, grouping the samples according to the season and the days in milk production. The samples were processed through a spectrophotometric enzymatic methodology for the determination of MUN. An analysis of variance was carried out for the groups by climatic season, while for the days in milk a quantitative description was made. Results. The MUN varied according to the season of the year $(p<0.05)$, the values being higher during the rainy season. The MUN was

Como citar (Vancouver).

Tarazona ML, Andrade BR, Archila BO. Variación del nitrógeno ureico en leche según la temporada del año y días en leche. Rev MVZ Córdoba. 2021; 26(3):e2012. https://doi.org/10.21897/rmvz.2012 
lower during the first 60 DIM in most of the conformed groups and the peaks appeared from 180 DIM. The lowest MUN values according to the days in milk were determined in the first 60 days in most of the conformed groups, likewise, the main peaks were determined from 180 DIM. Conclusions. The MUN varied according to the season, being higher in rains, the MUN was lower during the first 60 DIM in most of the groups formed and the peaks appeared from 180 DIM.

Keywords: Dairy cows; protein; nutritional balance; urea; energy (Sources: DeCS, CAB).

\section{INTRODUCCIÓN}

La urea es el producto final de la conversión del nitrógeno y tiene dos orígenes, el primero es el amonio no usado, el cual es formado en el rumen y convertido en urea en el hígado; y el segundo, es el catabolismo de los aminoácidos en el cuerpo. Cuando la urea se encuentra en la sangre, toma el nombre de nitrógeno ureico sanguíneo (NUS) $(1,2,3)$.

Debido a su pequeño tamaño, las moléculas de urea son capaces de atravesar las membranas celulares (incluidas las de la glándula mamaria) y llegar hasta la leche, allí toma el nombre del nitrógeno ureico en leche (NUL), que constituye entre el 2.5 a $3 \%$ del total del nitrógeno lácteo. Por tanto, NUL es derivado principalmente del NUS y está fuertemente relacionado con éste $(4,5,6)$.

En bovinos, el NUL se relaciona con el consumo de proteína cruda, la degradabilidad de esta en la ración y el balance energético de la vaca. Cuando el nivel de producción aumenta, la relación proteína-energía de la comida igual, resultando en un incremento del amonio que no puede ser usado por las bacterias ruminales, lo cual produce una elevación en la producción de urea, seguida por un incremento de las concentraciones de NUS y el NUL. La suplementación en la dieta con nitrógeno tipo urea afecta la síntesis microbiológica ruminal y la síntesis hepática de urea en el ganado lechero $(2,3)$.

EI NUL puede variar por condiciones externas como la temporada de muestreo, el tipo de muestreo, el almacenamiento de la muestra, su procesamiento, el estado de lactancia de la hembra, edad al parto, cantidad de partos y nivel de producción $(7,8,9)$. Además, varían entre rebaños, grupos de vacas y vacas del mismo grupo (10).

El NUL es un indicador importante y efectivo para establecer el estatus de nitrógeno ureico en vacas lecheras, pues esta herramienta permite identificar fácilmente una subalimentación o sobrealimentación de proteína $(11,12)$. Su monitoreo es importante porque el NUL está relacionado con desórdenes de tipo reproductivo, e incremento de células somáticas, así como con un aumento en la excreción de nitrógeno con un impacto ambiental negativo $(10,13,14,15)$.

En Colombia, pocos estudios se relacionan con el análisis de NUL en rebaños lecheros $(16,17)$, y con la variación del NUL con respecto a los días en leche (DEL) en las condiciones de clima tropical del país. Se evidencia, en la literatura científica, la ausencia de estudios sobre las variaciones de NUL asociadas a las temporadas climáticas y a los días en producción de leche de las vacas de los rebaños nacionales. El municipio de Duitama se caracteriza por su producción láctea, haciendo parte del denominado "cordón lechero boyacense".

Por ello, el objetivo del presente trabajo fue determinar la variación del NUL según la temporada (épocas de lluvia y seca), y los días en leche de vacas lecheras de dos ganaderías del municipio de Duitama en el altiplano boyacense de Colombia.

\section{MATERIALES Y MÉTODOS}

Tipo y sitio de estudio. Se realizó un muestreo por conveniencia, descriptivo, cuantitativo. El estudio fue desarrollado en dos granjas del municipio de Duitama- Boyacá; el cual presenta una temperatura promedio de $14^{\circ} \mathrm{C}$ en época de lluvia y $18^{\circ} \mathrm{C}$ en la época seca (18). Las granjas incluidas en el estudio distan una de la otra en dos kilómetros y presentan condiciones ambientales similares de temperatura, pluviosidad, pasturas y reciben agua del mismo río.

Animales incluidos en el estudio. Se seleccionaron 112 vacas lecheras de la raza Holstein, entre dos y tres partos, y entre 5 y 305 DEL. De la granja uno se seleccionaron 50 animales, con un peso promedio de $460.6 \pm 55.7$ $\mathrm{kg}$ y un promedio de producción de $17.5 \mathrm{~kg}$ de leche/día/vaca. Por otro lado, 62 animales de 
la segunda granja, con un peso promedio de $452.5 \pm 42.3 \mathrm{~kg}$ y un promedio de producción de $18.3 \mathrm{~kg}$ leche/día/vaca.

Sólo vacas con condiciones óptimas de salud fueron incluidas en el estudio, eso significó, sin cojeras, ni enfermedades respiratorias, reproductivas y de la ubre. Se consideraron sanas de la glándula mamaria con conteos menores a 200.000 células $/ \mathrm{mL}$. El análisis de conteo de células somáticas se analizaba en cada muestreo. La presencia de conteos de células somáticas superiores a lo estipulado constituía el descarte de la muestra para ese análisis.

Alimentación. Los animales pastaban en praderas con Kikuyo (Cenchrus clandestinum). Recibieron durante cada ordeño (a.m. y p.m.) $2 \mathrm{~kg}$ de alimento balanceado comercial, con valores de humedad de $13 \%$, proteína cruda del $14 \%$, cenizas de $10 \%$, grasa de $2.5 \%$ y fibra cruda de $25 \%$.

Ordeño. En ambas granjas, el ordeño es mecánico y se realizaba dos veces al día, la primera a las 3:00 a.m. y la segunda a las 14:00 p.m.

Muestreo. Las muestras fueron recolectadas previo al inicio del ordeño y posterior a la limpieza de los pezones, la cual fue realizada con solución yodada al 10\% (20), que se recolectaron de los cuatro pezones fueron mezcladas. Una muestra se consideró la mezcla de una deyección por cada uno de los pezones en cada uno de los ordeños, es decir, la muestra de la mañana fue almacenada en una nevera a una temperatura de $6^{\circ} \mathrm{C}$ para ser mezclada con la muestra de la tarde y luego fueron transportadas a una temperatura de $4^{\circ} \mathrm{C}$ hasta su procesamiento $(7,19)$. Dos muestras por mes fueron tomadas durante todo el período de estudio, para un total de 5.376 muestras en total, sin embargo, se excluyeron 475 muestras debido a aumentos en el conteo de células somáticas por encima de 200.000 células $/ \mathrm{mL}$, por lo tanto, el número total de muestras incluidas en el estudio fue de 4.901.

Procesamiento. Las muestras fueron analizadas el mismo día de su recolección en el Laboratorio de Análisis de la Calidad de Leche y Control de Mastitis en la Universidad Pedagógica y Tecnológica de Colombia. El análisis de NUL se usó la metodología enzimática espectofotométrica usando un equipo Mindray BS120 utilizando el kit UREA de LAB TEST a una longitud de onda de 450nm (21). Además, el conteo de células somáticas fue realizado con un Fossomatic ${ }^{\mathrm{TM}} 7$ basado en citometría de flujo.
Recolección de datos de la finca. Las fincas incluidas en el estudio organizaban los datos con respecto a los días en lactancia y el número de partos en una base de datos del programa Excel ${ }^{\circledR}$ para Microsoft 2010. Los datos de los años 2018 y 2019 fueron tomados e incluidos dentro del análisis estadístico efectuado.

Categoría de análisis. Los trimestres fueron seleccionados de acuerdo a los datos del Instituto de Hidrología, Meteorología y estudios ambientales para la zona (18), donde se separan las temporadas climatológicas en dos, con dos períodos para cada una: seca, la cual comprende el primer período de enero a marzo (la cual se denominará en adelante S1 y de acuerdo al año, 18 o 19, y un segundo período de octubre a diciembre (S2); y lluvias (la cual se denominará en adelante LL1 y de acuerdo al año 18 o 19), el cual comprende el primer período de abril a junio, y el segundo desde julio a septiembre (LL2), la diferencia entre esos dos períodos por cada época y entre épocas es la intensidad de las lluvias.

Los datos con respecto a los DEL se agruparon a partir de los primeros 5 días en leche hasta el día 30 , y a partir de allí, los datos fueron agrupados cada 30 días hasta el día 330.

Análisis de datos. Para el análisis de la variación a través de las temporadas, un promedio de NUL de ambas granjas fue realizado, para cada trimestre, sin discriminación de los DEL y se realizó un análisis de varianza para cada temporada por año, incluyendo como factor de bloqueo las fincas, usando la comparación de las medias de la mínima diferencia significativa del procedimiento Fisher, con un nivel de confianza del 95\%, usando el software Statgraphics Centurion ${ }^{\circledR}$, versión para Windows 10 . Por otro lado, se realizó un análisis descriptivo del promedio de NUL para los grupos de DEL (cada 30 días) por cada uno de los trimestres conformados.

\section{RESULTADOS}

Se determinaron diferencias estadísticamente significativas entre los trimestres incluidos para los dos años evaluados. Los menores valores de NUL fueron identificados en los periodos S1$18, \mathrm{~S} 2-18$ y S2-19. Por otro lado, los mayores valores se encontraron en la temporada LL1-18, LL2-18, LL2-19. 
Los resultados del promedio de NUL para cada trimestre durante los dos años se muestran en la figura 1.

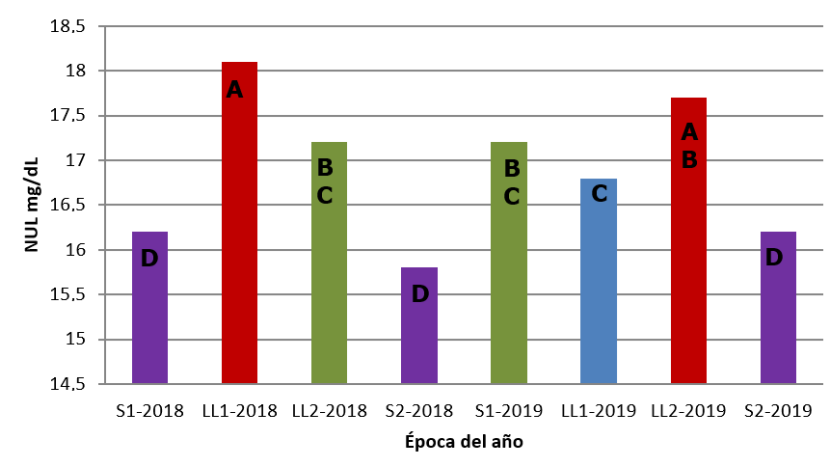

Figura 1. Promedios de NUL según la temporada del año (épocas de lluvia y seca) de vacas lecheras en dos ganaderías en el altiplano boyacense de Colombia. Columnas con letras diferentes indica diferencias estadísticamente significativas $(p<0.05)$.

Se presentó un efecto estadísticamente significativo de la época del año sobre la concentración de NUL $(p<0.05)$ en el primer año (2018) los mayores resultados fueron obtenidos en la época de lluvia, con diferencias estadísticas entre grupos, cuando éste se compara con el 2019, no muestra el mismo comportamiento, encontrando que el primer trimestre de la época seca fue estadísticamente el mismo comparado con el segundo periodo de lluvias en el 2018 ( $p>0.05)$, y el primer periodo de lluvia del 2019 tiene un menor valor comparado con el primer trimestre del mismo año y con el mismo período en el 2018 ( $p>0.05$ ). Sin embargo, la mayoría de los trimestres de la época seca tuvieron el mismo porcentaje promedio para el NUL $(p>0.05)$.

Variación de NUL de acuerdo con los DEL. La figura 2 muestra el año 2018, para el primer trimestre (S1-18) el promedio de NUL tiene tres incrementos, el primero entre los 61 y 90 DEL igual que LL1; el segundo entre los 151 y $180 \mathrm{DEL}$, y el tercero entre los 211 y $240 \mathrm{DEL}$, lo interesante es la excepción del período LL2, los otros trimestres tuvieron el casi el mismo comportamiento para el último incremento (211 y $240 \mathrm{DEL})$.

Los trimestres S2 y LL2 tuvieron su primer incremento entre los 31 y $60 \mathrm{DEL}$, pero el LL2 incrementa de nuevo entre los 121 y 150 días, y el último aumento importante, junto con la LL1 fueron entre los 301 y 330 días, mientras que los períodos S1 y S2 disminuyeron en el mismo período.

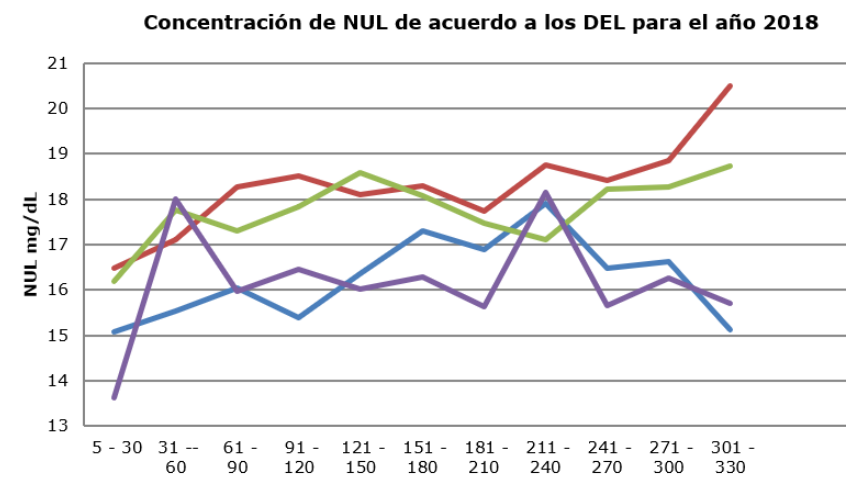

Figura 2. Promedio de NUL según los días en de vacas lecheras en dos ganaderías en el altiplano boyacense de Colombia de acuerdo con los DEL en el 2018. Línea azul: S1-2018. Línea roja: LL1-2018. Línea verde: LL2-2018. Línea púrpura: S2-2018.

Comparado con los datos del 2018, el promedio de NUL de acuerdo con los DEL fue diferente (Figura 3). La época S1 únicamente tiene dos incrementos, el primero entre los 91-120 DEL junto con la S2, mientras que los períodos LL1 y LL2 disminuyeron el mismo período. La S1I tiene el segundo incremento entre los $241 \mathrm{y}$ $270 \mathrm{DEL}$, mientras que el resto de los períodos disminuyeron. Por otro lado, las épocas LL1 y LL2 tuvieron su primer incremento entre los 61 y $90 \mathrm{DEL}$, con un segundo incremento entre los 151 y $210 \mathrm{DEL}$, con una disminución entre los 241 y 270 DEL, y allí la LL2 aumentó mientras que la LL1 disminuyó.

Concentración de NUL de acuerdo a los DEL para el año 2019

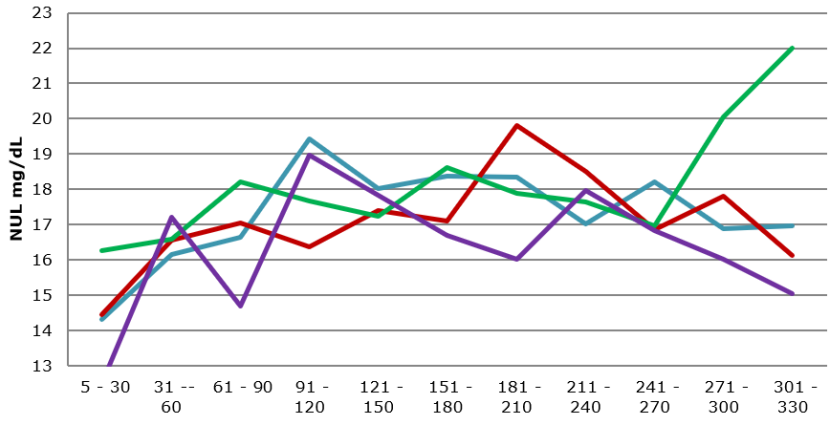

Figura 3. Promedio de NUL según los días en de vacas lecheras en dos ganaderías en el altiplano boyacense de Colombia de acuerdo con los DEL en el 2019. Línea azul: S1-2019. Línea roja: LL1-2019. Línea verde: LL2-2019. Línea púrpura: S2-2019. 


\section{DISCUSIÓN}

Los valores normales de NUL varían de acuerdo con la bibliografía consultada, por ejemplo, estudios en Brasil, mencionan valores ideales de NUL en vacas lecheras entre 10 y $14 \mathrm{mg} / \mathrm{dL}$, siendo así, los valores determinados en este estudio están por encima de este rango, indicando posibles manejos nutricionales deficientes en los dos rebaños evaluados. E incluso los valores presentados aquí, son elevados con respecto a los reportados por los mismos investigadores en la misma raza (10). Sin embargo, no fue posible relacionar los resultados presentados debido a falta de información relacionada con el manejo de los potreros, la composición y variación de la dieta suministrada a los animales, y el comportamiento productivo general de los animales dentro de la granja.

Por otro lado, para el caso colombiano, investigaciones presentan una clasificación de NUL en donde valores por encima de 18 $\mathrm{mg} / \mathrm{dL}$ pueden generar alteraciones de tipo reproductivo, valores entre 15 y $18 \mathrm{mg} / \mathrm{dL}$ existe una subutilización del nitrógeno, y que los valores óptimos que debería manejar todo rebaño son entre 12 y $15 \mathrm{mg} / \mathrm{dL}$ (22). Los resultados muestran que ninguno de los valores reportados para ninguno de los trimestres se encuentra en los valores óptimos para la producción y reproducción. Y que para los DEL los únicos que muestran mayoritariamente estar en el grupo óptimo es en los primeros $30 \mathrm{DEL}$. Incluso, existen reportes de valores normales de NUL en ciertos rebaños de raza Holstein se ubican por encima de $40 \mathrm{mg} / \mathrm{dL}$ (23).

Para las condiciones del departamento de Antioquia (Colombia) se determinó un crecimiento constante de la concentración de NUL hasta los 115 DEL alcanzando un valor de $17.75 \mathrm{mg} /$ $\mathrm{dL}$ (17). Estos resultados son contrarios a la mayoría de los comportamientos encontrados a lo largo de los dos años de estudio, debido a que los únicos dos periodos en donde el crecimiento en la concentración de NUL fue constante fue en las épocas LL1-2018 y S1-2019, las demás concentraciones revelaron crecimientos y disminuciones sin patrón visible durante los primeros $120 \mathrm{DEL}$, el valor máximo determinado en este estudio se dio en la época S1-2019 con un resultado de $19.42 \mathrm{mg} / \mathrm{dL}$.

Posteriormente, los investigadores evidenciaron una disminución constante hasta los 190DEL hasta llegar a una concentración de $(17.40 \mathrm{mg} /$
$\mathrm{dL}$ ); la única época en la que este comportamiento se evidenció en la S2-2019, las demás épocas presentaron comportamientos inconstantes de aumentos y disminuciones que son contrarios a lo reportado por Henao et al (17). Por último, los investigadores mencionaron un aumento constante de la concentración hasta el día 300 hasta alcanzar una concentración de $(18.50 \mathrm{mg} /$ $\mathrm{dL}$ ), este mismo comportamiento se evidenció en las épocas LL1-2018, LL2-2018 y LL2-2019.

Algunas investigaciones mencionan que el contenido de NUL en granjas holandesas varía de acuerdo con el tipo de alojamiento en el cual se encuentran los animales, aquellos de granjas más pequeñas y menos organizadas presentan un valor mucho más alto $(5,24)$, y menciona además, que en esas regiones en los meses de verano el contenido de urea es significativamente mayor (24). Debido a las condiciones climatológicas de este país, es importante destacar que el régimen alimenticio durante este período cambia, ofreciendo a los animales alimentos más digeribles y con mejores concentraciones de nutrientes, lo cual afecta el comportamiento del NUL en estos rebaños, debido a la condición tropical de Colombia no existe propiamente la época de verano, sino dos períodos secos, en los cuales, debido a la poca tecnificación de las granjas nacionales no se ofrecen alimentos altamente aprovechables como los ofrecidos en Europa.

En un estudio efectuado en Brasil en época lluviosa (enero a mayo), sometieron a diversas hembras lactantes de raza "Mantiqueira" a diferentes cantidades de proteína bruta en la dieta (450, 600 y 750 gramos PB), y analizando hasta $60 \mathrm{DEL}$ encontraron valores de NUL de $14.15 \mathrm{mg} / \mathrm{dL}, 16.50 \mathrm{mg} / \mathrm{dL}$ y $20.71 \mathrm{mg} / \mathrm{dL}$ respectivamente (25). Estos valores son similares a los encontrados en los mismos trimestres de esta investigación. De igual manera, los resultados son similares a los determinados para los grupos menores de 60 DEL de este estudio, mostrando un comportamiento similar a pesar de la distancia geográfica y de las condiciones climáticas entre los dos países.

Se ha determinado también, fuentes de variación del NUL en vacas lecheras de diversas razas, encontrando que sin importar la raza, el número de partos y la cantidad de leche producida, los valores de NUL aumentarán en los períodos lluviosos, mientras que los menores valores se presentarán en la época de verano (10). Estos cambios son explicados debido 
a las alteraciones estructurales que sufren las pasturas, particularmente en la porción digestible, la cual disminuye drásticamente.

Se ha establecido que el NUL tiene una disminución en los primeros $60 \mathrm{DEL}$ y un pico significativo principalmente cerca de la mitad de la lactancia debido a factores de cantidad de consumo alimenticio, porque en el puerperio la ingesta disminuye drásticamente en los animales, y a medida que va avanzando la lactancia este consumo se normaliza y, por tanto, los valores de NUL empiezan a aumentar (10). Comportamientos similares a lo reportado fueron determinados en la presente investigación, en donde la mayoría de los menores valores se presentaron en los primeros 60 DEL y se muestran picos de aumento a partir de día 181 y no a partir de los 151 como otros estudios $(7,10)$.

Un resultado contradictorio fue encontrado para los valores de NUL en el mundo, en donde los investigadores determinaron que para ganado Holstein los mayores valores de NUL ocurrían en el verano $(28 \mathrm{mg} / \mathrm{dL}$ ) comparado con los meses de invierno ( $24.95 \mathrm{mg} / \mathrm{dL})$, aduciendo estos resultados a los ingredientes con los cuales fueron preparadas las dietas que recibieron los animales con respecto a la temporada de cosecha (11).
Por otro lado, en Brasil, se efectuó un estudio similar en búfalos de acuerdo a los meses del año determinando para el período lluvioso (agosto a enero) un valor promedio mayor $(18.56 \mathrm{mg} / \mathrm{dL})$ que en el período seco (febrero a julio) donde se determinó un valor promedio de $15.91 \mathrm{mg} / \mathrm{dL}$ (26); estos valores muestran un comportamiento similar a lo reportado en este estudio en donde los mayores valores para el NUL se determinaron para el período lluvioso en general en los dos años, con valores similares a los propuestos en el estudio mencionado.

En conclusión, los mayores valores del NUL se determinaron, a través de los dos años de estudio, en la temporada de lluvias con respecto a la temporada seca $(p<0.05)$. El NUL fue menor durante los primeros 60 DEL en la mayoría de los grupos conformados y los picos se presentaron a partir de los $180 \mathrm{DEL}$.

\section{Conflicto de intereses}

Todos los autores declaran que no existe ningún conflicto de intereses para la publicación del presente manuscrito.

\section{REFERENCIAS}

1. Hossein N, Ardalan M. Estimation of genetic parameters for milk urea nitrogen and its relationship with milk constituents in Iranian Holsteins. J Liv Sci. 2011; 135(23):274-281. http://dx.doi.org/10.1016/j. livsci.2010.07.020.

2. Mucha S, Strandberg E. Genetic analysis of milk urea nitrogen and relationships with yield and fertility across lactation. J Dairy Sci. 2011; 94(11):5665-5672. https://doi. org/10.3168/jds.2010-3916.

3. Jin D, Zhao S, Zheng N, Bu D, Beckers $Y$, Wang $\mathrm{J}$. Urea nitrogen induces changes in rumen microbial and host metabolic profiles in dairy cows. J Liv Sci. 2018; 210(4):104-110. https://doi.org/10.1016/j. livsci.2018.02.011.
4. Sawa A, Boguchi M, Krężel S. Effect of some factors on relationships between milk urea levels and cow fertility. Arch Tierz. 2011; 54(5):468-476. https://doi.org/10.5194/ aab-54-468-2011.

5. Ruska D, Junkens D. Crude Protein and Non-protein Nitrogen Content in Dairy Cow Milk. Proc Latv Univ Agr. 2014; 32(1):36-40. https://doi.org/10.2478/plua-2014-0011.

6. Wernersbach $\mathrm{H}$, Souza J, Assis A, Campos $\mathrm{S}$, Queiroz A, Diniz R, et al. Variáveis ruminais, concentração de uréia plasmática e excreções urinárias de nitrogênio em vacas leiteiras alimentadas com concentrado processado de diferentes formas. R Bras Zootec. 2006; 35(3): 1236-1241. https://doi.org/10.1590/ S151635982006000400039. 
7. Arunvipas P, Dohoo I, VanLeeuwen J, Keefe G. The effect of non-nutritional factors on milk urea nitrogen levels in dairy cows in Prince Edward Island, Canada. Prev Vet Med. 2003; 59(1-2):83-93. https://doi. org/10.1016/s0167-5877(03)00061-8.

8. Rzewuska K, Strabel T. Genetic parameters for milk urea concentration and milk traits in Polish Holstein-Friesian cows. J Appl Genet. 2013; 54(4):473-482. https://doi. org/10.1007/s13353-013-0159-8.

9. Imran $M$, Nasser $T$, Qamer $M$, Babar I, Naveed $M$. Effect of increasing dietary metabolizable protein on nitrogen efficiency in Holstein dairy cows. 2017. J Anim Sci. 30(5):660-665. https://doi.org/10.5713/ ajas. 16.0564 .

10. Droska M, Ferreira D, Horst J, Valloto A, Rossi $\mathrm{P}$, Almeida R. Sources of variation in milk urea nitrogen in Paraná dairy cows. R Bras Zootec. 2012; 41(3):692-697. https://doi. org/10.1590/S1516-35982012000300032.

11. El Shewy T, Kholif S, Morsy T. Determination of milk urea nitrogen for the Egyptian cattle fed the summer and winter diets. J Amer Sci. 2010; 6(12):382-384. http://www. jofamericanscience.org/journals/am-sci/ am0612/44 3303am0612 382 384.pdf.

12. Kgole M, Visser C, Banga C. Environmental factors influencing milk urea nitrogen in South African Holstein cattle. S Afr J Anim Sci. 2012; 42(5):459-463. http://dx.doi. org/10.4314/sajas.v42i5.3.

13. Silva V, Rangel H, Galvão J, Urbano S, Borba $L$, Novaes $L$, et al. Influence of somatic cell count in the composition of girolando cow's milk in tropical zone. Trop Sub Agro. 2016; 19(2):101-107. http://www. revista. ccba.uady. $\mathrm{mx} / \mathrm{ojs} /$ index.php/TSA/article/ view/2193.

14. Kananub S, VanLeeuwen J, Arunvipas P. Association between milk urea nitrogen and first service conception in smallholder dairy farms under heat and humidity stress. Vet World. 2018; 11(11):16041608. https://dx.doi.org/10.14202/ vetworld.2018.1604-1608.
15. Kananub S, Jawjaroensri W, VanLeeuwen J, Stryhn H, Arunvipas P. Exploring factors associated with bulk tank milk urea nitrogen in Central Thailand. Vet World. 2018; 11(5):642-648. https://dx.doi. org/10.14202/vetworld.2018.642-648.

16. Galvis $R$, Correa $H$, Barrientos $S$, Muñoz $Y$. Efecto de niveles crecientes de nitrógeno no proteico dietario en vacas lactantes sobre las concentraciones de metabolitos nitrogenados en orina, sangre y leche. Rev Fac Nal Agr Medellín. 2011; 64(2):61916198. https://revistas.unal.edu.co/index. php/refame/article/view/29407.

17. Henao A, Múnera O, Herrera A, Agudelo J, Cerón M. Lactose and milk urea nitrogen: fluctuations during lactation in Holstein cows. R Bras Zootec. 2014; 43(9):479484. http://dx.doi.org/10.1590/S1516$\underline{35982014000900004}$

18. Instituto de Hidrología, Meteorología y Estudios Ambientales. Boletín meteorológico. 2019. [Consultado diciembre de 2019]. URL disponible en: http://www.ideam.gov.co/ web/tiempo-y-clima/tiempo-clima

19. Marques $P$, Machado $P$, Coldebella A, Dagher L, Oliveira K, Mazza P. Validation of models for predicting milk urea nitrogen concentrations, estimating dry matter intake by the NRC (2001). R Bras Zootec. 2012; 41(5):1271-1277. https://doi.org/10.1590/ $\underline{\text { S1516-35982012000500026 }}$

20. Tarazona L, Villate J, Andrade R. Bacterial and fungal infectious etiology causing mastitis in dairy cows in the highlands of Boyacá (Colombia). Rev Med Vet Zoot. 2019, 66(3):208-218. https://doi:10.15446/ rfmvz.v66n3.84258

21. Sánchez J. Nitrógeno ureico en leche: importancia, determinación y relación con otros componentes lácteos. Nut Anim Trop. 2016; 10(2):20-37. https://doi. org/10.15517/NAT.V10I2.26111.

22. Cerón $M$, Henao $A$, Múnera $O$, Herrera $A$, Díaz $A$, Parra $A$, et al. Concentración de nitrógeno ureico en leche, interpretación y guía práctica. 2014. Ed. Universidad de Antioquia. https://revistas.udea.edu.co/ index.php/biogenesis/article/view/326014 
23. Nozad S, Gholi A, Moghadam G, Asri S, Babapour A, Ramin A. Relationship between blood urea, protein, creatinine, triglycerides and macro-mineral concentrations with the quality and quantity of milk in dairy Holstein cows. Vet Res For. 2012; 3(1):5559. https://www.ncbi.nlm.nih.gov/pmc/ articles/PMC4312820/

24. Fagan $P$, Cabreira $C$, Calixto $M$, Simili $M$, Tadeu G. Fatores ambientais e de manejo sobre a composição química do leite em granjas leiteiras do Estado do Paraná, Brasil. Acta Sci. 2010; 32(3):309-316. https://doi. org/10.4025/actascianimsci.v32i3.8570.
25. Soussa C, Valvasori E, Peixoto K, Fontolan V. Concentrações de nitrogênio na dieta, no sangue e no leite de vacas lactantes no período pós-parto. R Bras Zootec. 2006; 35(1):258-263. https://www.rbz.org.br/ pt-br/article/concentracoes-de-nitrogeniona-dieta-no-sangue-e-no-leite-de-vacaslactantes-no-periodo-pos-parto/.

26. Do Nascimento A, Diocleciano A, Chaves T, Monteiro T, Lima D. Concentration of urea nitrogen in buffalo milk during different seasons of the year in northeastern Brazil. Rev Caat. 2013. 26(3):99-104. https://periodicos.ufersa.edu.br/index.php/ caatinga/article/view/2569. 